\title{
Challenges in Retention of People living with HIV/AIDS, along Continuum of Care and Treatment in Pakistan
}

\section{Abdul Baseer Khan Achakzai* and Pasha S}

National Institute of Health, Chak Shehzad, Park Road, Islamabad, 44000, Pakistan

*Corresponding author: Abdul Baseer Khan Achakzai, National Institute of Health, Chak Shehzad, Park Road, Islamabad, 44000, Pakistan, Tel: +92-51-9255096; Email: achakzaibk@gmail.com

Received date: December 24, 2015; Accepted date: January 14, 2016; Published date: January 21, 2015

Copyright: (c) 2016 Achakzai ABK, et al. This is an open-access article distributed under the terms of the Creative Commons Attribution License, which permits unrestricted use, distribution, and reproduction in any medium, provided the original author and source are credited.

Keywords: Retention in HIV-care; Pre-ART care; Continuum of care; Antiretroviral treatment

\section{Description}

Pakistan currently faces a 'concentrated epidemic' among two of the 'key populations' including people who inject drugs (PWID) and transgender sex workers [1]. However, HIV prevalence among general population still remained low i.e., $0.09 \%$ as per the latest UNAIDS Spectrum estimates [2].

ART program, in Pakistan was rolled in 2004 and gradually expanded with establishment of 20 ART Clinics throughout the country. Antiretroviral (ARV) drugs and CD4 tests are freely available to PLHIV at ART clinics [3]. PLHIV records are maintained in preART and ART enrollment registers and subsequently reported through a web-based MIS, designed for ART Clinics to the national database [4].

Retention of people living with HIV/AIDS (PLHIV) during HIVcare and treatment is vital for success of antiretroviral therapy (ART) interventions. ART health care services are planned to reduce HIVrelated morbidity and mortality, associated with HIV infection and prevention of appearance of drug resistance [4]. PLHIV may be lost along the care and treatment cascade pathway firstly; HIV testing to enrolment into care services; secondly, enrolment in care to ART eligibility; thirdly, eligibility to initiation of ART, and lastly, initiation to lifelong ART [4].

Effective HIV treatment, as prevention requires clients to be tested, aware of their status, linked and retained in care and virally suppressed. We conducted a cross-sectional systematic review and meta-analysis of MIS data from ART clinics, to obtain magnitude of patient's loss from diagnosis to suppressed viral load [5]. This review aimed to quantify patient's losses, along the continuum of care \& treatment and review possible interventions to decrease attrition.

Spectrum data for 2015 estimated 93,925 PLHIV, in Pakistan [2]. Among 24,525 clients [3], screened HIV positive, up to end June 2015, $45 \%(11,038)$, were enrolled in HIV care and assessed for their ART eligibility. Meanwhile 5,019 PLHIV [4] i.e., 9.03\% of those actual in need of ART, as per the Spectrum/EPP estimates, were initiated with ART [2]. Data on viral suppression couldn't be extracted of the clinical records for PLHIV maintained at the ART Clinics. However, an analysis of 15 PLHIV charts from an ART clinic at Karachi showed that of the people retained on ART, more than $80 \%$ had a viral load of $<1000$ copies/ml after 12 months on ART.
The cascade pathway indicated an enormous 'testing gap'; $26.1 \%$ clients screened positive of total estimated. Fear of stigma and discrimination, disclosure affecting their livelihoods and limited availability of testing facilities, as major contributing factors [6]. Other major losses included 55\% diagnosis to registration; followed by $15.5 \%$ lost to follow-up during retention in ART [3]. Key contributing factors included; number and geographical location of ART clinics, inadequate pre-ART care, high level of stigmatization, perceived lack of staff confidentiality, long distance to treatment sites, lack of health insurance \& $\mathrm{CHBC}$ services and non-existence of ART treatment literacy [6].

To conclude despite expansion of ART program, retention of PLHIV in HIV-care is still remained a key challenge. The study suggested need to adopt strategies such as 'point of care' (POC) CD4testing; PLHIV tracking for those who are 'lost to follow-up' (LTFU); adaptation of new WHO strategy of initiating ART at CD4-count cutoff level of 500 cells $/ \mathrm{mm}^{3}$ to reduce number of patients in Pre-ART care [7]; decentralizing standalone ART services by integrating HIVcare at district level care facilities, i.e., District Headquarters hospitals, antenatal clinics, and TB program.

The study was limited by lack of appropriate understanding for some concepts (e.g., linkage to care, loss to follow up) and overlap in the different steps of the continuum of care was observed.

We acknowledge contribution of ART physicians for their support and cooperation of Association of People Living with HIV/AIDS (APLHIV) and without their support the study would have never been completed.

\section{References}

1. Pakistan Global AIDS Response Progress Report (2015) National Integrated Biological and Behavioral Surveillance (IBBS). NACP.

2. UNAIDS (2014) Strengthening HIV Estimates: EPP/Spectrum 2015.

3. www.nacp.gov.pk

4. Ulett KB, Willig JH, Lin HY, Routman JS, Abroms S, et al. (2009) The therapeutic implications of timely linkage and early retention in HIV care. AIDS Patient Care STDS 23: 41-49.

5. Rosen S, Fox MP, Gill CJ (2007) Patient retention in antiretroviral therapy programs in sub-Saharan Africa: a systematic review. PLoS Med 4: e298.

6. PashaSK (2013) Country Report On National Research Study On HIV Community Access To Treatment, Care And Support Services In Pakistan.

7. WHO (2013) Consolidated Guidelines on the Use of Antiretroviral Drugs for Treating and Preventing HIV Infection. 